Australia, changes in autopsy numbers coincided with changes in Coroners Acts. However, changes in autopsy numbers per year were also observed in without modifications to Coroners Acts. The findings suggest that forensic pathology autopsy workload can be influenced by changes in Coroners Acts and by changes relating to operating polices within individual jurisdictions.

\section{GAME OVER: COCAINE AND VIDEO GAMES AS CONTEMPORARY RISK FACTORS FOR PULMONARY EMBOLISM}

Lena Quinto, Rebecca Irvine

Forensic Medicine and Coroner's Court Complex, Lidcombe, NSW, Australia

Pulmonary embolism (PE) is a life-threatening condition in which emboli, often from the legs or pelvis lodge in the pulmonary vasculature causing impairment of gas exchange and right ventricular strain. Virchow's triad of hypercoagulable state, endothelial activation and venous stasis is a framework for understanding risk factors. Yet, some risk factors seem deceptively benign or esoteric.

The case described is of a 33-year-old male who was found suddenly and unexpectedly deceased. According to family, he would play video games for extended periods of time including overnight. Autopsy examination showed the body of an obese male with the presence of bilateral pulmonary thromboemboli. Toxicological analysis showed the presence of metabolites of cocaine. There are multiple factors that contributed to PE in this individual. Obesity may have contributed to PE through its association with venous stasis. Extended periods of time engaging in video games has been associated with PE through venous stasis and a hypercoagulable state if dehydrated. Cocaine is prothrombotic by causing endothelial injury with subsequent chemokine activation. Cocaine may cause a hypercoagulable state as a result of increased haematocrit. We discuss these factors and highlight the need to consider non-traditional risk factors.

\section{INCREASED PREVALENCE OF SODIUM NITRITE SUICIDES IN SOUTH AUSTRALIA}

Lilli Stephenson $^{1}$, Steven Wills ${ }^{2}$, Corinna van den Heuvel ${ }^{1}$, Melissa Humphries $^{3}$, Roger W. Byard ${ }^{1,2}$

${ }^{1}$ Adelaide Medical School, University of Adelaide, Adelaide, SA, Australia; ${ }^{2}$ Forensic Science South Australia, Pathology Group, Adelaide, SA, Australia; and ${ }^{3}$ School of Mathematical Sciences, University of Adelaide, Adelaide, SA, Australia

Sodium nitrite $\left(\mathrm{NaNO}_{2}\right)$ is a water-soluble, white-yellow coloured crystalline powder with broad industrial applications. ${ }^{1}$ In Australia, it has also been used to humanely control growth of feral pig populations. ${ }^{2}$ While several cases of accidental consumption have been reported in the literature, sodium nitrite has recently emerged as an increasingly popular method of pharmacological suicide. This method has been recommended in The Peaceful Pill Handbook ${ }^{3}$ with additional information and 'suicide kits' available online. Between 2000 and 2019, 10 cases of intentional sodium nitrite ingestion were identified from records at Forensic Science South Australia and the National Coronial Information System. All 10 cases occurred in the latter 3 years of the study period. All decedents had a history of depression or chronic illness and demonstrated characteristic post-mortem signs of methemoglobinaemia (blue-grey post-mortem hypostasis and dark brown discolouration of blood and internal organs). This study highlights a significant emerging public health issue. Awareness of emerging fatal substance abuse trends, clinical symptoms and post-mortem signs by health professionals and forensic pathologists is critical to effectively identifying these cases at presentation and autopsy.

\section{References}

1. Benowitz N. Nitrates and nitrites. In: Olsen K, editor. Poisoning and Drug Overdose. $7^{\text {th }}$ ed. New York: McGraw Hill, 2018; 339-40.

2. Shapiro L, Eason C, Bunt C, et al. Efficacy of encapsulated sodium nitrite as a new tool for feral pig management. $J$ Pest Sci 2016; 89: 489-95.

3. Nitschke P, Stewart F. The Peaceful Pill eHandbook. Blaine, WA: Exit International, 2018.

\section{CTNNB1 EXON 3 MUTATIONS IN DESMOID AND OTHER TUMOURS}

Raewyn Billings ${ }^{1}$, Katie A. Ashton ${ }^{1}$, Kathy Tucker ${ }^{2}$, Andrew Ziolkowski ${ }^{1}$, Mary McPhillips ${ }^{1}$, Michael Hipwell ${ }^{1}$, Rodney J. Scott ${ }^{1}$

${ }^{1}$ Department of Molecular Medicine, NSW Health Pathology, John Hunter Hospital, NSW, Australia; and ${ }^{2}$ Hereditary Cancer Centre, NSW Health Pathology, Prince of Wales Hospital, NSW, Australia

Desmoid tumours are rare soft tissue tumours. The tumours are a locally invasive monoclonal fibroblastic proliferation leading to a variable clinical course. Abnormal functioning of the Wnt/APC/ Beta catenin pathway accounts for a large proportion of desmoid tumours. There are currently two genetic subtypes: sporadic desmoid tumours resulting from somatic CTNNB1 mutations in exon 3 and desmoid tumours associated with germline pathogenic variants in APC. APC and CTNNB1 mutations are thought to be mutually exclusive. Mutations in CTNNB1 are also associated with molecular subtypes of other tumours including hepatocellular adenomas and childhood medulloblastomas.

Using Sanger sequencing, we searched for hotspot mutations in exon 3 of CTNNB1 using a case series of 13 patients with desmoid tumours. 11/13 patients harboured CTNNB1 mutations. We also assisted in the histopathological diagnosis of two other tumours with aberrant IHC staining (hepatocellular adenoma and medulloblastoma), both harbouring CTNNB1 mutations.

Hepatocellular adenomas with beta catenin mutations are associated with a high risk of malignant transformation and WNTactivated medulloblastomas are associated with a very good prognosis. The molecular subclassification of desmoid tumours and other tumours, combined with histopathology, assists with understanding the genetic basis of disease, tailoring treatment options and provides informative prognosis. Our laboratory offers CTNNB1 testing. 